\title{
Blocking of Pseudomonas aeruginosa and Chromobacterium violaceum lectins by diverse mammalian milks
}

\author{
K. D. Zinger-Yosovich, D. Iluz, D. Sudakevitz, and N. Gilboa-Garber ${ }^{1}$ \\ The Mina and Everard Goodman Faculty of Life Sciences, Bar-Ilan University, Ramat-Gan 52900, Israel
}

\begin{abstract}
Pseudomonas aeruginosa and Chromobacterium violaceum morbid and mortal infections are initiated by bacterial adherence to host-cell receptors via their adhesins, including lectins (which also contribute to bacterial biofilm formation). Pseudomonas aeruginosa produces a galactophilic lectin, PA-IL (LecA), and a fucophilic (Lewis-specific) lectin, PA-IIL (LecB), and C. violaceum produces a fucophilic (H-specific) lectin, CV-IIL. The antibiotic resistance of these bacteria prompted the search for glycosylated receptor-mimicking compounds that would function as glycodecoys for blocking lectin attachment to human cell receptors. Lectins PA-IL and PA-IIL have been shown to be useful for such glycodecoy probing, clearly differentiating between human and cow milks. This article describes their usage, together with CV-IIL and the plant lectin concanavalin A, for comparing the anti-lectin-dependent adhesion potential of diverse mammalian milks. The results show that the diverse milks differ in blocking (hemagglutination inhibition) and differential binding (Western blots) of these lectins. Human milk most strongly inhibited the 3 bacterial lectins (with PA-IIL superiority), followed by alpaca, giraffe, and monkey milks, whereas cow milk was a weak inhibitor. Lectin PA-IL was inhibited strongly by human, followed by alpaca, mare, giraffe, buffalo, and monkey milks, weakly by camel milk, and not at all by rabbit milk. Lectins PA-IIL and CV-IIL were also most sensitive to human milk, followed by alpaca, monkey, giraffe, rabbit, and camel milks but negligibly sensitive to buffalo and mare milks. Plant lectin concanavalinA, which was used as the reference, differed from them in that it was much less sensitive to human milk and was equally as sensitive to cow milk. These results have provided important information on the anti-lectin-dependent adhesion potential of the diverse milks examined. They showed that human followed by alpaca, giraffe, and Rhesus monkey milks efficiently blocked the binding of both the galactophilic
\end{abstract}

Received May 14, 2009.

Accepted September 10, 2009.

${ }^{1}$ Corresponding author: garben@mail.biu.ac.il and fucophilic (>mannophilic) pathogen lectins. The results also proved the advantage of isolated pathogenic bacterial lectins as superb probes for unveiling bacterial adhesion-blocking glycodecoys. The chosen milks or their polymeric glycans might be implicated in blocking lectin-dependent adhesion of antibiotic-resistant pathogens leading to skin, eye, ear, and gastrointestinal infections.

Key words: bacterial lectin, Chromobacterium violaceum, milk anti-adhesion, Pseudomonas aeruginosa

\section{INTRODUCTION}

The opportunistic pathogens Pseudomonas aeruginosa and Chromobacterium violaceum are involved in human and animal morbid and mortal antibioticresistant infections (Sirinavin et al., 2005; Lim et al., 2009; Strateva and Yordanov, 2009). Pseudomonas aeruginosa is one of the leading nosocomial pathogens worldwide (Strateva and Yordanov, 2009), infecting patients suffering from cystic fibrosis, AIDS, chronic granulomatous diseases, burns, ventilator-associated pneumonia, renal transplantations, and aplastic anemia as well as patients recovering from heart surgery. It can aggressively attack any organ, including lungs, spleen, liver, gut, kidneys, brain, heart, eyes, ears, and skin (Gilboa-Garber et al., 1997), leading to severe damage that might terminate in lethal sepsis (Long et al., 2008). Chromobacterium violaceum is also involved in invasive, aggressive infections, but those are rarer, being restricted in distribution to tropical and subtropical regions. Chromobacterium violaceum has also been reported to be associated with abscesses of lungs, liver, spleen, and skin and to be able to attack every organ and lead to fatal bacteremia (Sirinavin et al., 2005; Lim et al., 2009). Infections by these bacteria are initiated following their adherence to the target cells by means of adhesins, including lectins. Pseudomonas aeruginosa produces a galactophilic lectin, PA-IL (LecA), displaying P-system and B combined with I blood group specificity (Gilboa-Garber et al., 1994) and a fucophilic (more than mannophilic) lectin, PA-IIL (LecB) (Gilboa-Garber, 1982; Chemani et al., 2009), which preferentially binds to Lewis epitopes, especially 
Le $^{\mathrm{a}}$ (Mitchell et al., 2002; Wu et al., 2006). These $2 P$. aeruginosa lectins bind to the same animal organs that are attacked by the intact bacterium (Gilboa-Garber et al., 1997). Chromobacterium violaceum also possesses a fucophilic, more than mannophilic, lectin, CV-IIL, which is structurally homologous to PA-IIL but differs from it in its preferential blood group $\mathrm{H}$ epitope affinity (Zinger-Yosovich et al., 2006).

The resistance of $P$. aeruginosa to antibiotics (Strateva and Yordanov, 2009) prompted the search for means that would hamper its lectin-dependent adhesion to its target-cell surface receptors. Inhibition of pathogenic bacterial lectin activity by glycans blocks lectin-dependent bacterial adhesion to their target host cells for infection establishment (according to the anti-adhesion strategy for prevention and treatment of antibiotic-resistant bacterial infections). Many synthetic compounds simulating PA-IIL-binding target cell receptors have been produced. Examination of their PA-IIL-binding affinities has shown that glycan clusters [oligosaccharides, dendrimeric polysaccharides, and branched glycoproteins (GP)] are the strongest inhibitors (Imberty et al., 2008; Johansson et al., 2008). Use of such compounds was shown to abrogate the PA-IILdependent P. aeruginosa biofilm production (Johansson et al., 2008).

The anti-adhesion strategy functions widely in nature. It is most powerful in the protection of neonates against infections, as exemplified by feeding newborn mammals with maternal milks (composed of mammarygland products and serum components; Jensen, 1995). The milks supply nutrients, enzymes, growth factors, hormones, and glycans. The latter play a prebiotic role, promoting bifidobacteria-dominant colonic microflora (Coppa et al., 2006), and function as glycodecoys, protecting the newborns against infections (Newburg, 2009).

Human milk contains galactosylated and fucosylated (Fuco1-2/3/4/6) oligosaccharides and GP. Other animal milks are also generally rich in galactose $(\mathbf{G a l})$ or N-acetylglucosamine, as in the compound N-acetyllactosamine and other $\beta$-galactosylated polymers, but exhibit a relatively poor repertoire of the Fuco1-2/3/4bearing oligosaccharides and GP. The Gal $\beta$ glycosidic linkage is an important structural element because the human intestine lacks enzymes able to hydrolyze it (with the exception of lactose decomposition by lactase). The indigestible $\beta$-galactosaccharides exhibit anti-infective functions by contributing to both prebiotic and immunomodulating activities (Coppa et al., 2006; Boehm and Stahl, 2007) and to anti-adhesive activity as receptorsimulating decoys that competitively inhibit microbial adhesion to their target cells (Newburg, 2009). Similar prebiotic effects have also been attained with artificial mixtures of short-chain galactooligosaccharides and long-chain fructooligosaccharides (galactooligosaccharides and fructooligosaccharides in a ratio of 9:1, respectively). These were shown to increase counts of adult and infant fecal bifidobacteria with reduction of pathogens and improvement of fecal $\mathrm{pH}$ and composition and to modulate immune cell interactions (Vos et al., 2007; van Hoffen et al., 2008).

Human-milk glycans were reported to inhibit the adhesion to human cells of Vibrio cholerae (Holmgren and Lindbhad, 1983), Streptococcus pneumoniae and Hemophilus influenzae (Andersson et al., 1986), Campylobacter jejuni (Ruiz-Palacios et al., 2003), and bacterial toxins such as Escherichia coli heat-stable enterotoxin (Cleary et al., 1983) and Shiga and Shiga-like toxins (Newburg et al., 1992). Human-milk fucosylated blood group $\mathrm{H}$ epitope-specific Fuco1-2Gal $\beta$-bearing oligosaccharides and GP were shown to protect suckling mice from the heat-stable enterotoxin of $E$. coli (Cleary et al., 1983) and prevent adherence of C. jejuni (Ruiz-Palacios et al., 2003) and clinical isolates of Candida albicans (Brassart et al., 1991) to human cells. Cravioto et al. (1991) found that fucosylated residues of human-milk secretory IgA and oligosaccharides inhibited the adhesion of enteropathogenic E. coli to HEp-2 cells, and Strömqvist et al. (1995) demonstrated human-milk glycan efficacy in blocking Helicobacter pylori. Additional examples of the contribution of human-milk glycans to the prevention of bacterial and fungal adhesion to cells, both in vitro and in vivo, have been recently reviewed by Newburg (2009). Concomitantly, Hong et al. (2009) have described the impressive phenomenon that the majority of newborns born to HIV-1-infected mothers are protected from becoming HIV infected despite the fact that breastfeeding is the predominant postnatal transmission route for HIV-1 infection in children. This report is in contrast to previous ones showing that breastfeeding did not protect newborns from HIV infection and that breastfed newborns became HIV infected much more readily than those receiving formula. The solution could be treatment of the milks for HIV elimination. The human-milk oligosaccharides $(0.5-1.0 \mathrm{~g} / 100 \mathrm{~mL})$ or GP that carry at least one Lewis receptor-simulating epitope might function as glycodecoys, competing with HIV-1 GP 120 envelope protein for DC-SIGN binding, inhibiting HIV1 capture by its DC-SIGN receptor and its subsequent transfer to CD $4^{+}-\mathrm{T}$ lymphocytes (Naarding et al., 2005; Boehm and Stahl, 2007). Only $\mathrm{Le}^{\mathrm{x}}$ in polymeric form or conjugated to protein could mimic the inhibitory activity, whereas free $\mathrm{Le}^{\mathrm{x}}$ epitope could not.

All these findings indicate the value of human-milk polymeric glycans for the prevention of lectin-dependent adhesion of antibiotic-resistant pathogens. Even cytomegalovirus-infected lactating mothers' milks, 
which had been reported to put premature infants at risk, were recommended to be used following antiviral treatment (Schleiss, 2006). However, because lectin-dependent adhesion may be accompanied by nonspecific adhesion, infection therapy has to include additional antibacterial elements to ensure its full success.

Application of the isolated PA-IL and PA-IIL lectins of $P$. aeruginosa for study of milk anti-adhesion activity showed that both were very sensitive to inhibition by human milk. Comparing human with bovine milks (using both hemagglutination-inhibition and Western blot tests) disclosed that human milk was much more efficient than cow milk in blocking these 2 lectins and that the fucophilic PA-IIL was a highly efficient probe for human milk (Lesman-Movshovich and Gilboa-Garber, 2003). Gustafsson et al. (2005) used antibodies and plant lectins for Western blot analyses of milks from diverse mammals in addition to human and cow, including goat, sheep (ewe), pig, horse (mare), dromedary camel, and rabbit. They reported that the precursor structure for blood group $\mathrm{H}$ type 1 was expressed in all species investigated, whereas only pig, dromedary camel, and rabbit milk proteins carried the $\mathrm{H}$ type 2 epitope, which is important for the inhibition of the adhesion of $H$. pylori, E. coli heat-stable toxin, and $C$. jejuni (Gustafsson et al., 2005). Pig milk was shown by them to contain the $H$. pylori-binding epitopes sialyl $\mathrm{Le}^{\mathrm{x}}$ and $\mathrm{Le}^{\mathrm{b}}$, which inhibit $H$. pylori binding both in vitro and in vivo (Gustafsson et al., 2006). Iwamori et al. (2008), who examined the binding of botulinum neurotoxin and cholera toxin to components of human, cow, and goat milks, found that the first toxin bound to GT1b and GQ1b in goat milk but not to any human or cow milk ganglioside, whereas the cholera toxin bound to GM1.

In our current research, the bacterial lectins PA-IL (galactophilic) and PA-IIL (fucophilic more than mannophilic), both of them from $P$. aeruginosa; CV-IIL (resembling PA-IIL in structure and carbohydrate specificity) of $C$. violaceum; and the plant lectin Con A (as a reference for a mannophilic lectin) have been used as probes for analyses of 13 different mammal milks. Inhibition of the hemagglutinating activities of the 3 pathogenic lectins by the diverse milks was examined to analyze their lectin-blocking potentials (content of efficient glycodecoys). Dialysis of the milks was performed for differentiation between the blocking activities of low-molecular weight (MW) oligosaccharides and high-MW GP. Western blotting was used for detection and identification of the high-MW GP. As galactophilic, fucophilic, and mannophilic probes, PAIL, PA-IIL, and CV-IIL represent not only themselves and the pathogens that bear them as adhesins, but also lectins of other pathogens that possess similar recep- tor specificities. Milk components, which block them, might also be used as decoys for preventing infections by the related pathogens.

\section{MATERIALS AND METHODS}

\section{Lectin Preparations}

The bacterial lectins used, PA-IL, PA-IIL, and CVIIL, were isolated from cell extracts of $P$. aeruginosa ATCC 33347 and C. violaceum (Bergonzini) ATCC 12472, which were purchased from the American Type Culture Collection (ATCC, Manassas, VA), as described earlier (Gilboa-Garber, 1982; ZingerYosovich et al., 2006). Concanavalin A (Con A) was purchased from Sigma-Aldrich Co. (St. Louis, MO). The $P$. aeruginosa lectins PA-IL and PA-IIL and the C. violaceum lectin CV-IIL were purified by affinity chromatography and quality controlled by SDS-PAGE analysis with Coomassie Brilliant Blue staining, as described by Gilboa-Garber (1982) and by ZingerYosovich et al. (2006).

\section{Milks}

Human milks were obtained from healthy mothers (volunteers) in the second to the fourth month following delivery. Buffalo, cow, goat, and sheep (ewe) milks were purchased from food markets. The other milks were obtained from domestic, farm, zoo, and safari animals during their active lactation period from $2 \mathrm{wk}$ following delivery. The animals were milked manually by D. Iluz or in his presence. Some of the animals were treated with oxytocin to increase their milk secretion. The milk volumes obtained were generally 10 to 100 $\mathrm{mL}$, depending on animal size and milk secretion. The animals examined included alpaca (Vicugna or Lama pacos), buffalo (Bubalus bubalis), camel (one-humped, Camelus dromedarius), cow (Bos taurus), deer (fallow deer, Dama dama), dog (Canis familiaris), giraffe (Giraffa camelopardalis), goat (white and black ones, Capra aegagrus hircus), horse (mare, Equus ferus caballus), monkey (Rhesus monkey, Macaca mulatta), rabbit (Oryctolagus cuniculus), and sheep (ewe, Ovis aries). Each sample was centrifuged $(10,000 \times g)$ for $10 \mathrm{~min}$, and the intermediate phase was carefully separated. A part of each milk sample was dialyzed overnight in saline (isotonic $0.85 \% \mathrm{NaCl}$ solution) for removal of lowMW components $(<10 \mathrm{kDa})$, and all the milk samples were stored at $-20^{\circ} \mathrm{C}$. The milk samples used for the hemagglutination-inhibition test were not diluted, nor were those used for the Western blottings, excluding 2 -fold dilutions of the alpaca and rabbit milks (because of their much greater protein concentration). 


\section{Hemagglutination and Its Inhibition Tests}

Papain-treated human type $\mathrm{O}(\mathrm{H})$ red blood cells (erythrocytes) were used. The erythrocytes (kindly obtained from the Magen David Adom National Blood Services in Israel) were washed 3 times with PBS $(0.025$ $M$ phosphate at $\mathrm{pH} 7.2$ in the isotonic saline solution) and then treated by $0.1 \%$ papain with $0.01 \%$ cysteine, as described previously (Gilboa-Garber, 1982). A 50- $\mu \mathrm{L}$ aliquot of each bacterial lectin preparation examined was serially diluted with $50 \mu \mathrm{L}$ of saline to produce a series of 2 -fold dilutions. Then, saline and $5 \%$ (vol/vol) suspension of the papain-treated erythrocytes in saline $(50 \mu \mathrm{L}$ each) were added to each tube. After $30 \mathrm{~min}$ at room temperature, the tubes were centrifuged for $30 \mathrm{~s}$ $(1,000 \times g)$, and hemagglutinating activity was examined as described previously (Gilboa-Garber, 1982).

In the hemagglutination-inhibition test, each examined milk was serially 2 -fold diluted in $50 \mu \mathrm{L}$ of saline, and then $50 \mu \mathrm{L}$ of the lectin solution (at greatest dilution leading to agglutination of all the erythrocytes in one large mass) was added to each tube. After $30 \mathrm{~min}$ at room temperature, $50 \mu \mathrm{L}$ of the $5 \%$ papain-treated human $\mathrm{O}(\mathrm{H})$ blood type erythrocyte suspension was added to each tube (Gilboa-Garber, 1982), and after another $30 \mathrm{~min}$, hemagglutination was examined as described earlier. Hemagglutination-inhibition intensity was represented by the number of 2 -fold dilutions $\left(\log _{2}\right.$ dilution $^{-1}$ ) in which no considerable hemagglutination was observed preceding its reappearance. The usage of both undialyzed and dialyzed preparations of each milk enabled differentiation between the contribution of the low-MW $(<10 \mathrm{kDa})$ oligosaccharides (present in the undialyzed preparations together with their GP) and the GP alone, which were retained following removal of the oligosaccharides by dialysis.

\section{Western Blot Analyses}

A $15-\mu \mathrm{L}$ sample of each milk without dilution (except the alpaca and rabbit milks, which were 2 -fold diluted because of the too-high protein levels) was used for this test so that both the discrete bands and the intensities of the interactions with the lectins would be represented. These samples were mixed 1:1 with sample buffer, boiled, and applied to the wells in $10 \%$ SDS-PAGE (at $140 \mathrm{~V}$ ) in Mini-Protean Cell 3 Electrophoresis (Bio-Rad, Hercules, CA), as described previously (Lesman-Movshovich and Gilboa-Garber, 2003). Following SDS-PAGE, the proteins were transferred to nitrocellulose $(0.45 \mu \mathrm{m}$; Bio-Rad $)$ membranes at $4^{\circ} \mathrm{C}$ for $2 \mathrm{~h}(85 \mathrm{~mA} / 40-50 \mathrm{~V})$ using the Minitrans-Blot Module (Bio-Rad). The membranes were incubated overnight in blocking buffer (PBS $0.02 \mathrm{M}, \mathrm{pH} 7.2$, con- taining $3 \%$ BSA and $0.05 \%$ Tween 20 ), exposed to the peroxidase-labeled lectins (about $1 \mu \mathrm{g} / \mathrm{mL}$, dissolved in the same blocking buffer with $0.1 \%$ Tween 20) at room temperature for $2 \mathrm{~h}$, and then thoroughly washed. The peroxidase reaction was visualized using enhanced chemiluminescence (Amersham International plc, Buckinghamshire, UK) and recorded onto photographic films. This test demonstrates the lectin interactions with the individual milk GP.

Controls with the labeled lectins in the presence of $0.3 M$ of the relevant blocking sugar (Gal for PA-IL and mannose for Con A, PA-IIL, and CV-IIL) in their reaction mixtures were used in parallel to rule out nonspecific (sugar-independent) lectin binding. Lectins PA-IIL, CV-IIL, and Con A were inhibited by $0.3 \mathrm{M}$ mannose. The fucophilic definition of PA-IIL and CVIIL is based on their outstandingly great fucose affinity (at nanomolar concentrations) versus significantly less (millimolar) mannose affinity, resembling Con A affinity to this saccharide.

\section{Statistical Evaluation}

The results of the hemagglutination and its inhibition tests were analyzed by Student's $t$-test. The data presented in the figures generally represent means \pm SEM of at least 5 experimental results for each lectin, except for a few cases in which the milk amounts were restricted, enabling only 3 experimental results.

\section{RESULTS}

The reference mannophilic plant lectin Con A interactions with all the milks were shown in both hemagglutination-inhibition and Western blot analyses (Figure 1), indicating that all of the milks contained glycosylated components including GP that bind to the Con A active site. In most of the examined milks, inhibition was associated with polymeric compounds (of MW greater than $10 \mathrm{kDa}$, which were retained after dialysis). As seen in their Western blots, they were mainly of the 40 to $80 \mathrm{kDa}$ GP fraction, encompassing lactoferrin (around $80 \mathrm{kDa}$ ), with an additional discrete band at around $33 \mathrm{kDa}$ in some of the milks. The giraffe, alpaca, and rabbit milks (giraffe more than alpaca and rabbit) were the strongest inhibitors, whereas human, ewe, and dog milks were the weakest (showing profoundly stained 60-80 kDa GP; Figure 1).

As seen in Figure 2, the blocking of $P$. aeruginosa galactophilic lectin PA-IL by the diverse milks was generally weaker than that of Con A and differed from it in the differential specificity spectrum [displaying the greatest sensitivity to human and mare (horse) milks in addition to the alpaca and giraffe milks, but no sensitivity to rabbit milk] and in stronger inhibitions by the 


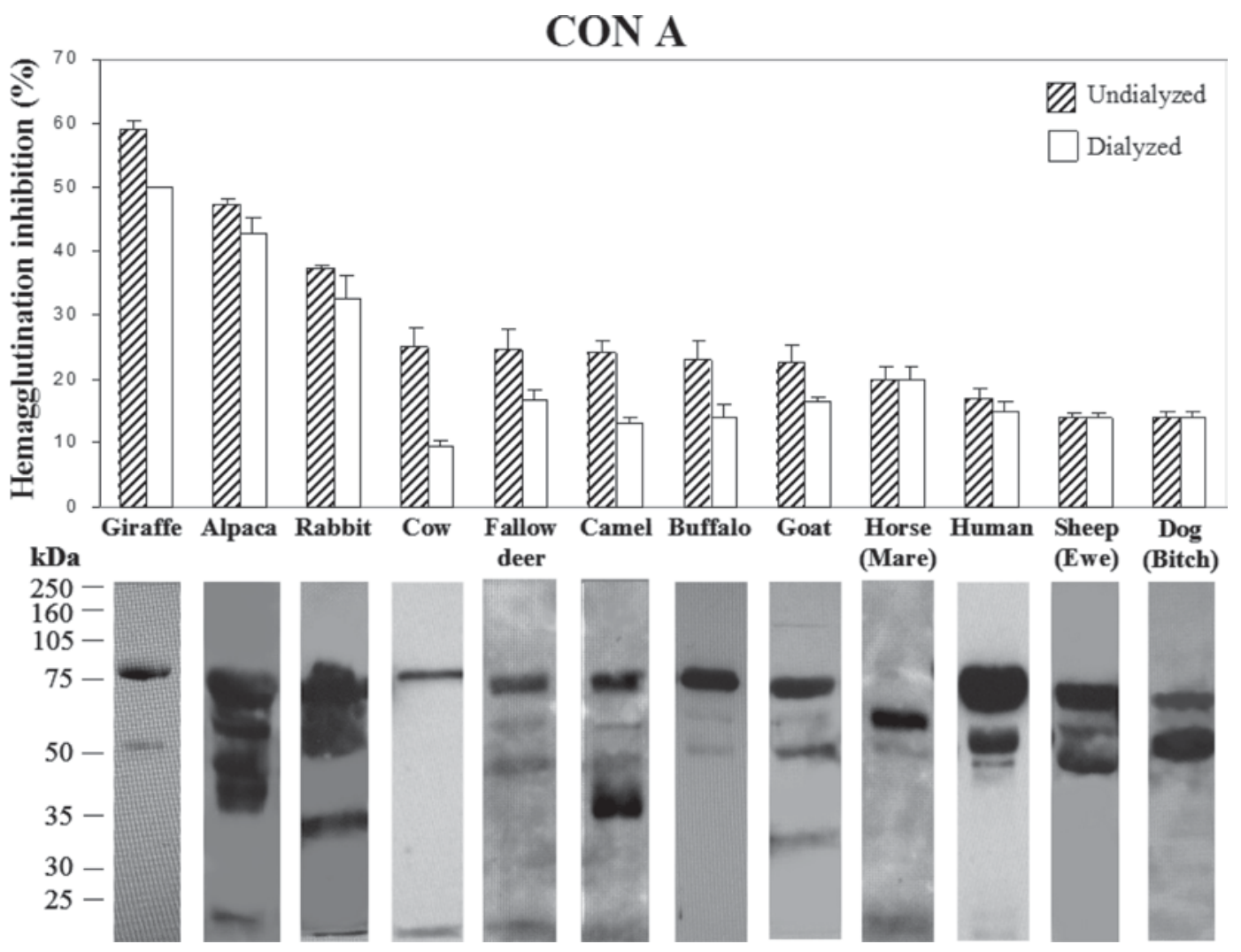

Figure 1. Interactions of the reference plant lectin concanavalin A (Con A) with the diverse milks (using undialyzed and dialyzed samples), as analyzed by hemagglutination-inhibition and Western blot tests. The hemagglutination-inhibition values are presented as the percentages of those obtained in the PA-IIL with undialyzed human milk combination $(100 \%)$. The Western blots were performed as described in the Materials and Methods section, using peroxidase-labeled Con A. The SEM of the second column, that of the dialyzed giraffe milk, is not noticeable in the figure (despite its inclusion in the data) because its dimension is very low (0.1) relative to the figure value scale.

milk low-MW oligosaccharides than by their GP. Lectin PA-IL was most strongly inhibited by undialyzed human, alpaca, horse, giraffe, and buffalo milks, followed by dialyzed human, alpaca, and horse milks. It was less sensitive to undialyzed cow, goat, sheep, and camel milks and was weakly inhibited by the dialyzed giraffe, buffalo, and all the other dialyzed milks, with no apparent interaction with rabbit milk components (Figure 2). The observation that the high inhibitions of PA-IL hemagglutinating activity by the giraffe and buffalo milks were almost totally abolished by removal of their low-MW components by dialysis was also supported by the Western blotting, showing considerable staining by the peroxidase-labeled PA-IL of only human, alpaca, and horse GP and very weak staining of the giraffe, buffalo, and cow Western blots. The absence of any interaction of rabbit milk with this lectin is in profound contrast to the considerable inhibitions of Con A, PAIIL, and CV-IIL by this milk and their strong staining of its GP.

Figure 3 shows that PA-IIL displayed prominent greatest sensitivity to human milk as compared with the other lectins, being inhibited by its glycans more strongly than by any of the other milks examined. This outstandingly high inhibition was due to both oligosaccharides (removable by dialysis) and GP (retained following dialysis), excluding the scant contribution of the rabbit oligosaccharides. As seen in the Western blots in Figure 3, PA-IIL interactions with the humanmilk GP were the strongest and of the widest range $(30-160 \mathrm{kDa})$. The alpaca and giraffe undialyzed milks followed human milk in their PA-IIL-inhibiting potencies. Their contribution was mainly associated with the oligosaccharide fraction (profoundly decreased following dialysis). Rabbit milk was the next inhibiting milk, outstanding in the absence of inhibition reduction by dialysis, which means that its inhibiting activity is confined to the GP glycans, which are apparent in the Western blots. Following the rabbit milk in decreasing inhibiting order was camel milk, with its active GP and oligosaccharides. Relatively lesser PA-IIL-inhibiting activities were observed in the sheep and dog milks (the latter exclusively associated with oligosaccharides); scant inhibitions were observed with the mare (horse), buffalo, and cow milks; and none was found with the fallow deer milk. 


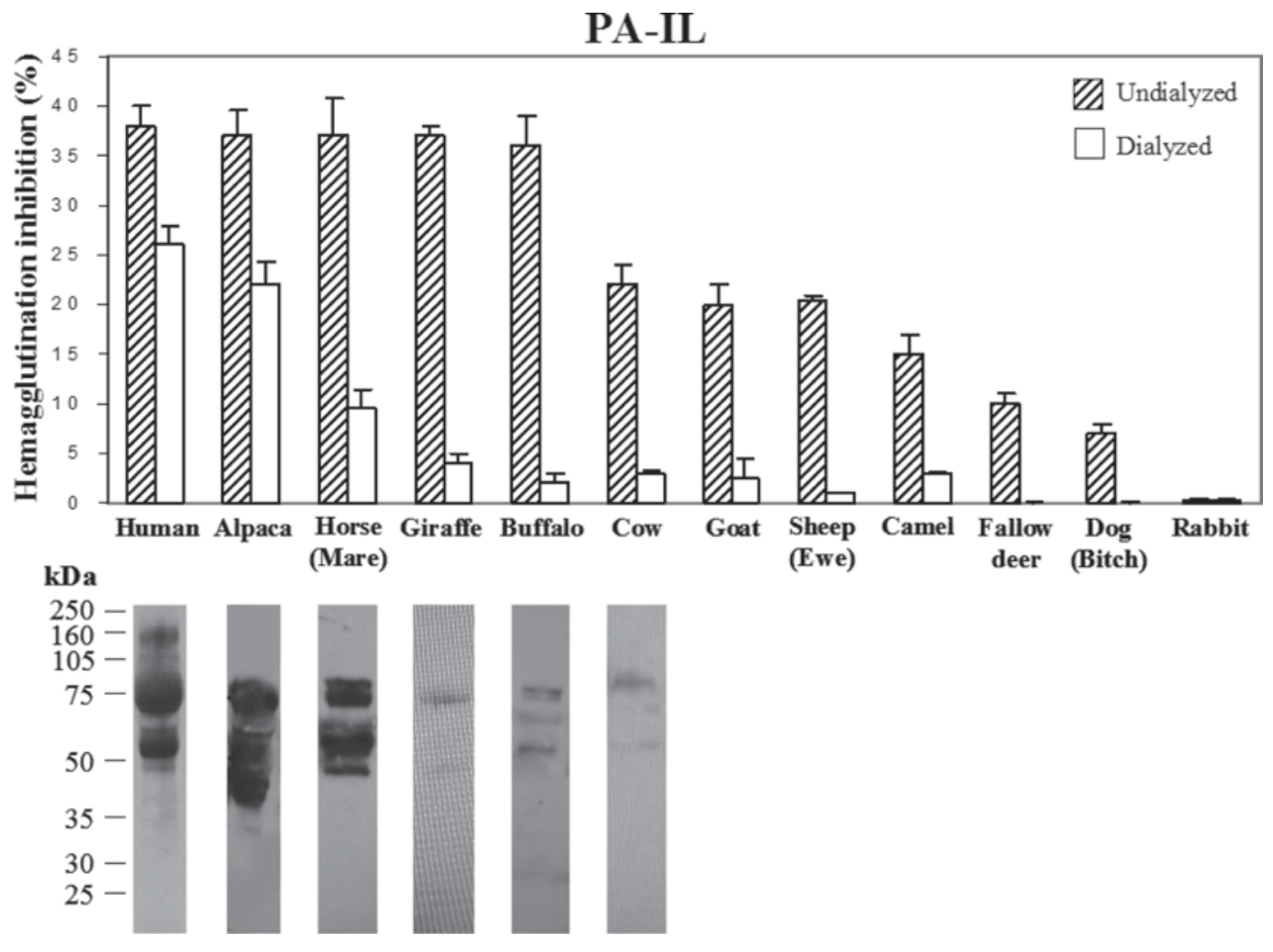

Figure 2. Interactions of the diverse milks with Pseudomonas aeruginosa galactophilic lectin PA-IL, as demonstrated by blocking of the lectin activity in the hemagglutination-inhibition test and staining of the electrophoretically separated glycoproteins of the 6 most potent lectinblocking milks in the Western blots by peroxidase-labeled PA-IL. The hemagglutination-inhibition values are presented as the percentages of those obtained in the PA-IIL with undialyzed human milk combination (100\%). The Western blots were performed as described in the Materials and Methods section.

Figure 4 shows that CV-IIL resembles PA-IIL in displaying prominent greatest sensitivity to human milk; followed by the group of giraffe, alpaca, camel, and rabbit; then the group of ewe, goat, dog, and mare (horse); followed by fallow deer and buffalo; and most weakly by cow milk. In addition to a few minor changes in the order, there were also differences in the relative contribution of the oligosaccharides versus GP to the lectin inhibitions.

Preliminary examination of Rhesus monkey milk (performed only once because of the minute amount available) indicated greatest inhibition of PA-IIL (50\% inhibition by undialyzed monkey's milk and $8 \%$ by dialyzed milk, related to $100 \%$ inhibition by undialyzed human milk) and a considerable inhibition of PA-IL (33\% inhibition by undialyzed milk and $2.5 \%$ by dialyzed milk) and of CV-IIL ( $22 \%$ inhibition by dialyzed milk), resembling the lectin-inhibition profile of the giraffe milk.

\section{DISCUSSION}

Milks of diverse mammals profoundly vary in their protein, lipid, and saccharide concentrations, includ- ing the milk disaccharide lactose levels. Primates are unique in having a greater proportion of calories as lactose and a lesser proportion as protein and fat [i.e., rabbit milk protein concentration is about 10 -fold greater than that of human milk, whereas human (and other primate) milk lactose level is 7 -fold greater than that of the rabbit milk; Table 1]. These and the milk glycan composition variations are determined by species specificity in association with genetic, evolutionary, and environmental factors. The milk glycans function in several scopes, including a) nutrition and prebiotic effects (Coppa et al., 2006; Boehm and Stahl, 2007), b) contribution to protection from infections by hindering lectin-dependent bacterial adhesion because of their function as glycodecoys that simulate the baby's cell receptors (Newburg, 2009), and c) immunomodulation exhibited in reduction of infant intestinal and respiratory infections and allergies (Vos et al., 2007; van Hoffen et al., 2008).

The resistance of milk glycans to digestion because of the lack of enzymes that hydrolyze them enables their effects along the intestinal tract. Human milk is rich in oligosaccharides that contain Gal $\beta$ glycosidic 


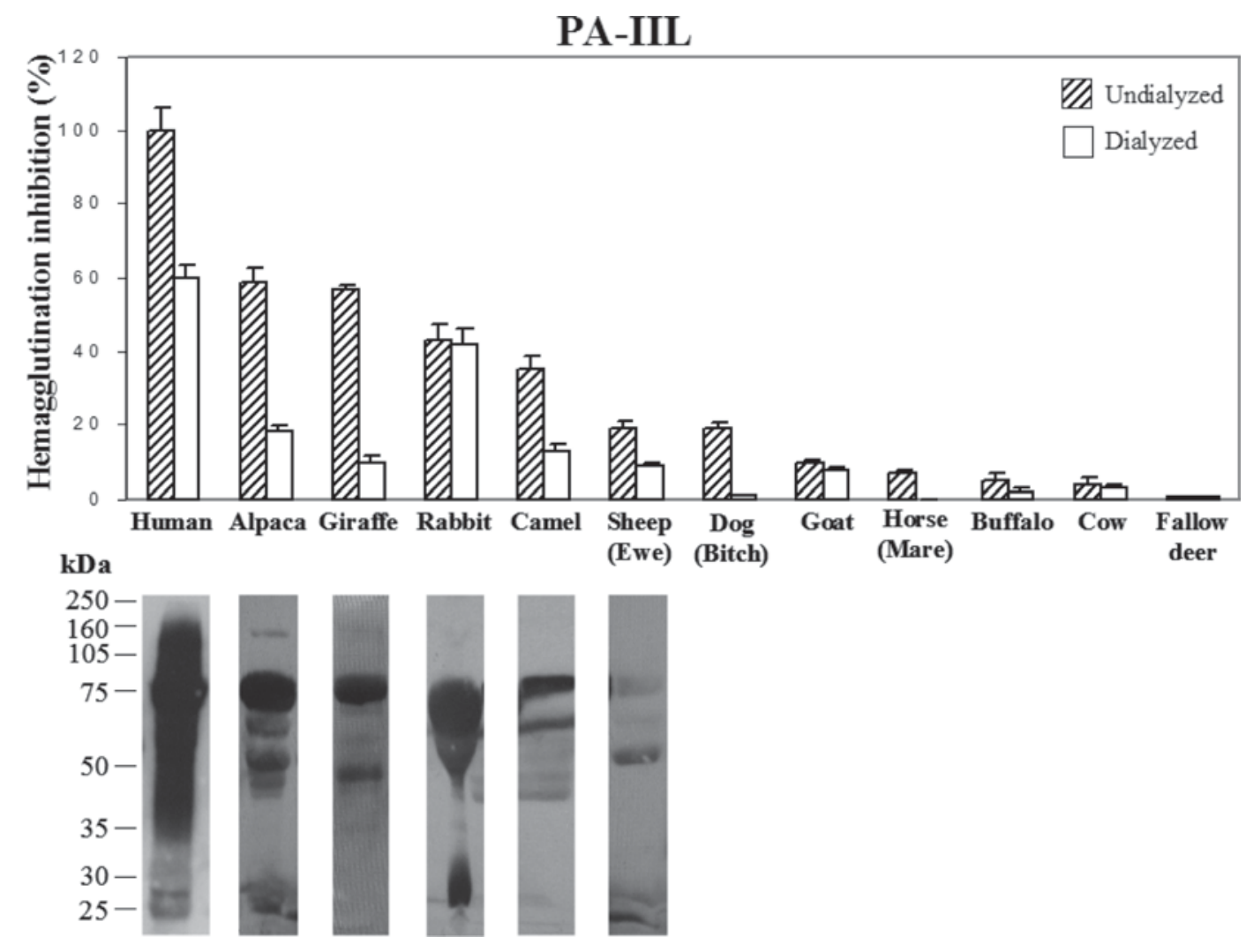

Figure 3. Interactions of the diverse milks with Pseudomonas aeruginosa fucophilic lectin PA-IIL, as demonstrated by blocking of the lectin activity in the hemagglutination-inhibition test and staining of the electrophoretically separated glycoproteins of the 6 most potent lectinblocking milks in the Western blots by peroxidase-labeled PA-IIL. The hemagglutination-inhibition values are presented as the percentages of those obtained in the PA-IIL with undialyzed human milk combination (100\%). The Western blots were performed as described in the Materials and Methods section.

linkage starting with lactose (Gal $\beta 1-4$ glucose) at the reducing end, to which there is a repetitive attachment of $[\mathrm{Gal} \beta 1-3 / 4 \mathrm{~N} \text {-acetylglucosamine } \beta 1-3]_{\mathrm{n}}=0-25$ in indigestible $\beta 1-3 / 6$ linkage (Boehm and Stahl, 2007). These oligosaccharides, with the exception of lactose, are indigestible by the human intestine. They can, therefore, pass the gastrointestinal tract and be used by health-promoting colonic bacteria that decompose them, positively affecting newborn intestinal flora (prebiotic effect; Coppa et al., 2006; Vos et al., 2007). In domestic animal milks, the concentration of such compounds is smaller by a factor of 10 to 100 , but their structure is similar to that of human milk or includes other galactooligosaccharidic structures $[\mathrm{Gal} \beta 1-]_{1-6^{-}}$ 3/4/6Gal $31-4$ glucose (goat, horse, sheep, cow; Boehm and Stahl, 2007).

The milk glycans that resemble the receptor epitopes of the pathogen-binding target cells might contribute to the protection of the newborn from infections by acting as glycodecoys that competitively hamper lectin-dependent pathogen binding to those receptors (Newburg, 2009). In addition to galactosylated structures, human milk contains fucosylated $\alpha 1-2 / 3 / 4$ oligosaccharides and GP that express blood group Lewis and $\mathrm{H}$ antigens that vary in accordance with the secretor and Lewis blood group status of the mothers, resulting in at least 4 groups of individuals. Animal-milk oligosaccharides have less Fuc $\alpha 1-2 / 3 / 4$ oligosaccharides, but in contrast to human milk, they contain both N-acetyl (Neu5Ac)and N-glycolyl-neuraminic acid (contributing to their anti-adhesion effect on uropathogenic $E$. coli). The Lewis and $\mathrm{H}$ antigens are receptors for clinical isolates of C. albicans (H epitope; Brassart et al., 1991) and $H$. pylori ( $\mathrm{Le}^{\mathrm{b}}$ and sialyl Le ${ }^{\mathrm{x}}$; Strömqvist et al., 1995), for enteropathogenic E. coli ( $\mathrm{H}$ type $1, \mathrm{Le}^{\mathrm{a}}$ and $\mathrm{Le}^{\mathrm{x}}$ ) and its heat-stable toxin (H types 1 and 2), and for C. jejuni (H type 2; Ruiz-Palacios et al., 2003; Newburg, 2009).

By virtue of its Lewis and $\mathrm{H}$-type fucosylated epitopes, human milk, in contrast to bovine milk, has been shown to most efficiently block $P$. aeruginosa fucophilic lectin PA-IIL, which displays very high affinity to the Lewis epitopes (Perret et al., 2005). Consequently, this lectin has been indicated as a most efficient probe for the selective detection of fucosylated Lewis-type glycans and for differentiation between human and bovine milk glycans (Lesman-Movshovich and Gilboa-Garber, 2003). A 


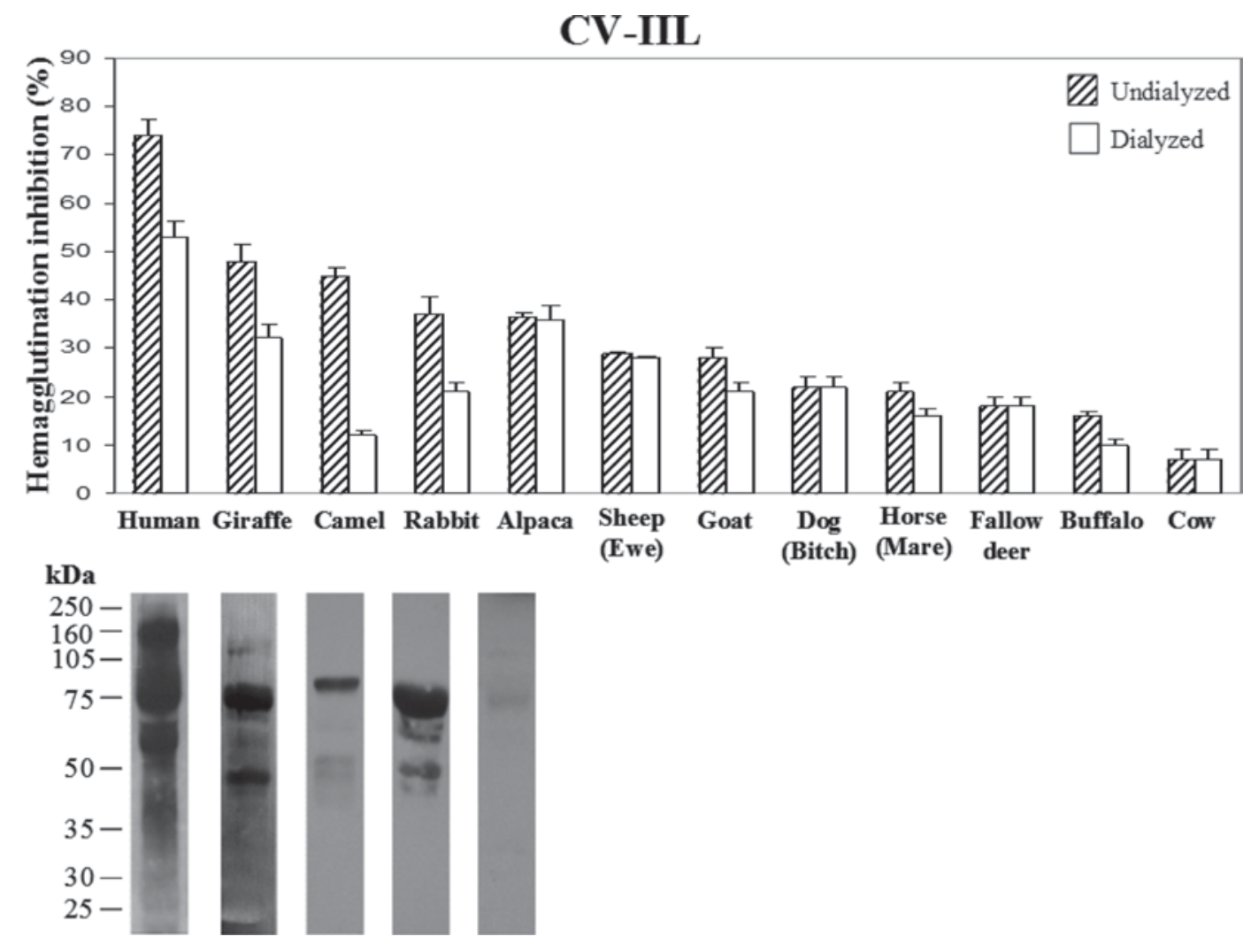

Figure 4. Interactions of the diverse milks with Chromobacterium violaceum fucophilic lectin CV-IIL, as demonstrated by blocking of the lectin activity in the hemagglutination-inhibition test and staining of the electrophoretically separated glycoproteins of the 5 most potent lectinblocking milks in the Western blots by peroxidase-labeled CV-IIL. The hemagglutination-inhibition values are presented as the percentages of those obtained in the PA-IIL with undialyzed human milk combination (100\%). The Western blots were performed as described in the Materials and Methods section.

similar difference between these 2 milks regarding their milk fat globule membranes was also recently described (Wilson et al., 2008).

The current study aimed at comparing the anti-adhesion potential of 13 mammalian milks using the human pathogenic $P$. aeruginosa galactophilic (PA-IL) and fucophilic (PA-IIL) lectins and C. violaceum fucophilic lectin (CV-IIL) as probes and adding the mannophilic plant lectin Con A as a reference. This work is closely related to that of Gustafsson et al. (2005) but differs from their work in 3 main aspects: 1) in analyzing the diverse mammalian milks, we used the pathogenic bac- terial lectins themselves as probes, whereas their probes were anti-blood group $\mathrm{ABH}$ and Lewis $\left(\mathrm{Le}^{\mathrm{a}}, \mathrm{Le}^{\mathrm{b}}, \mathrm{Le}^{\mathrm{x}}\right.$, sialyl $\mathrm{Le}^{\mathrm{a}}$, and sialyl $\mathrm{Le}^{\mathrm{x}}$ ) antibodies and several plant lectins; 2) we used hemagglutination-inhibition (blocking of the lectin activities) tests in addition to Western blot analyses; and 3) we added milks of alpaca, buffalo, deer, dog, giraffe, and monkey to their hitherto examined milk series that included woman, cow, goat, sheep (ewe), pig, horse (mare), dromedary camel, and rabbit. Gustafsson et al. (2005) demonstrated that the blood group precursor structure of $H$ type 1 is expressed in all species investigated, but the type 1 fucosylated car-

Table 1. Protein and lactose concentrations (\%) in the diverse mammalian milks (not including colostrum) based on data collected from Adkins et al. (2001); Farah (1993); Forsyth et al. (1975); Gustafsson et al. (2005); Jenness (1980); Jensen (1995); Kunz and Lonnerdal (1993); and Morin et al. $(1995)^{1}$

\begin{tabular}{|c|c|c|c|c|c|c|c|c|c|c|c|c|c|}
\hline Milk & Alpaca & Buffalo & Camel & Cow & $\begin{array}{c}\text { Fallow } \\
\text { deer }\end{array}$ & $\begin{array}{c}\text { Dog } \\
\text { (bitch) }\end{array}$ & $\begin{array}{l}\text { Sheep } \\
\text { (ewe) }\end{array}$ & Giraffe & Goat & Human & Horse (mare) & Monkey & Rabbit \\
\hline Protein & 8.0 & 4.0 & 3.0 & 3.0 & 10.0 & 7.0 & 5.5 & 6.0 & 3.3 & 1.0 & 2.5 & 2.0 & 12.0 \\
\hline Lactose & 6.5 & 5.0 & 4.0 & 4.7 & 2.4 & 4.0 & 6.0 & & 4.5 & 7.0 & 6.0 & 7.0 & 1.1 \\
\hline
\end{tabular}

${ }^{1}$ High protein and lactose values are marked in bold. 
bohydrate epitopes are found only on human, pig, and horse milk proteins, whereas the type 2 epitopes are present only on human, pig, dromedary camel, and rabbit milks (Gustafsson et al., 2006). The results of the current study showed that whereas the cow, goat, and sheep (ewe) milks are weak lectin inhibitors, the alpaca, giraffe, and monkey milks resemble those of dromedary camel and rabbit in being similar to human milk in their pathogenic bacterial lectin-blocking potential for the inhibition of the lectin-dependent adhesion of $H$. pylori, E. coli heat-stable toxin, and C. jejuni (Gustafsson et al., 2005).

\section{CONCLUSIONS}

Probing of 13 milks for pathogenic $P$. aeruginosa and C. violaceum lectin blocking showed that human milk was the most efficient, followed by alpaca, giraffe, and monkey milks. Lectin PA-IL was also strongly inhibited by mare and buffalo milks and PA-IIL and CV-IIL by rabbit and camel milks. Cow, deer, dog, goat, and sheep milks were weak inhibitors. The blocking milk glycans might be used as glycodecoys for abrogation of lectindependent pathogen anchoring to host cells in external (skin, ear, eye) and intestinal infections involving the 2 previously mentioned bacteria, as well as other antibiotic-resistant pathogens possessing similar lectins. Because there are also other adhesins and virulence factors, the lectin blocking should be accompanied by additional protective components ensuring infection abrogation.

\section{ACKNOWLEDGMENTS}

The authors express their gratitude to Sharon Victor and Ela Gindy (editorial and graphic units, Faculty of Life Sciences, Bar Ilan University, Ramat-Gan, Israel) for their important help with the editing and preparation of this manuscript and the graphic presentation. This work is part of the PhD thesis of K. D. ZingerYosovich at Bar-Ilan University, Israel.

\section{REFERENCES}

Adkins, Y., A. J. Lepine, and B. Lonnerdal. 2001. Changes in protein and nutrient composition of milk throughout lactation in dogs. Am. J. Vet. Res. 62:1266-1272.

Andersson, B., O. Porras, L. A. Hanson, T. Lagergard, and C. Svanborg-Eden. 1986. Inhibition of attachment of Streptococcus pneumoniae and Haemophilus influenzae by human milk and receptor oligosaccharides. J. Infect. Dis. 153:232-237.

Boehm, G., and B. Stahl. 2007. Oligosaccharides from milk. J. Nutr. 137:847S-849S.

Brassart, D., A. Woltz, M. Golliard, and J. R. Neeser. 1991. In vitro inhibition of adhesion of Candida albicans clinical isolates to human buccal epithelial cells by Fuc-alpha-1-2 Gal beta-bearing complex carbohydrates. Infect. Immun. 59:1605-1613.

Chemani, C., A. Imberty, S. de Bentzmann, M. Pierre, M. Wimmerova, B. P. Guery, and K. Faure. 2009. Role of LecA and LecB lectins in Pseudomonas aeruginosa-induced lung injury and effect of carbohydrate ligands. Infect. Immun. 77:2065-2075.

Cleary, T. G., J. P. Chambers, and L. K. Pickering. 1983. Protection of suckling mice from heat-stable enterotoxin of Escherichia coli by human milk. J. Infect. Dis. 148:1114-1119.

Coppa, G. V., L. Zampini, T. Galeazzi, and O. Gabrielli. 2006. Prebiotics in human milk: A review. Dig. Liver Dis. 38(Suppl. 2):S291-S294.

Cravioto, A., A. Tello, H. Villafan, J. Ruiz, S. del Vedovo, and J. R. Neeser. 1991. Inhibition of localized adhesion of enteropathogenic Escherichia coli to HEp-2 cells by immunoglobulin and oligosaccharide fractions of human colostrum and breast milk. J. Infect. Dis. 163:1247-1255.

Farah, Z. 1993. Composition and characteristics of camel milk. J. Dairy Res. 60:603-626.

Forsyth, I. A., P. D. Rossdale, and C. R. Thomas. 1975. Studies on milk composition and lactogenic hormones in the mare. J. Reprod. Fertil. Suppl. 23:631-635.

Gilboa-Garber, N. 1982. Pseudomonas aeruginosa lectins. Methods Enzymol. 83:378-385.

Gilboa-Garber, N., D. Avichezer, and N. C. Garber. 1997. Bacterial lectins: Properties, structure, effects, function and applications. Pages 369-398 in Glycosciences: Status and Perspectives. H. J. Gabius and S. Gabius, ed. Chapman \& Hall, Weinheim, Germany.

Gilboa-Garber, N., D. Sudakevitz, M. Sheffi, R. Sela, and C. Levene. 1994. PA-I and PA-II lectin interactions with the $\mathrm{ABO}(\mathrm{H})$ and $\mathrm{P}$ blood group glycosphingolipid antigens may contribute to the broad spectrum adherence of Pseudomonas aeruginosa to human tissues in secondary infections. Glycoconj. J. 11:414-417.

Gustafsson, A., A. Hultberg, R. Sjostrom, I. Kacskovics, M. E. Breimer, T. Boren, L. Hammarstrom, and J. Holgersson. 2006. Carbohydrate dependent inhibition of Helicobacter pylori colonization using porcine milk. Glycobiology 16:1-10.

Gustafsson, A., I. Kacskovics, M. E. Breimer, L. Hammarstrom, and J. Holgersson. 2005. Carbohydrate phenotyping of human and animal milk glycoproteins. Glycoconj. J. 22:109-118.

Holmgren, A. M. S., and M. Lindbhad. 1983. Receptor-like glycocompounds in human milk that inhibit classical and El for Vibrio cholerae cell adherence (hemagglutination). Infect. Immun. 39:147-154.

Hong, P., M. R. Ninonuevo, B. Lee, C. Lebrilla, and L. Bode. 2009. Human milk oligosaccharides reduce HIV-1-gp120 binding to dendritic cell-specific ICAM3-grabbing non-integrin (DC-SIGN). Br. J. Nutr. 101:482-486.

Imberty, A., Y. M. Chabre, and R. Roy. 2008. Glycomimetics and glycodendrimers as high affinity microbial anti-adhesins. Chemistry (Easton) 14:7490-7499.

Iwamori, M., K. Takamizawa, M. Momoeda, Y. Iwamori, and Y. Taketani. 2008. Gangliosides in human, cow and goat milk, and their abilities as to neutralization of cholera toxin and botulinum type A neurotoxin. Glycoconj. J. 25:675-683.

Jenness, R. 1980. Composition and characteristics of goat milk: Review 1968-1979. J. Dairy Sci. 63:1605-1630.

Jensen, R. G. 1995. Handbook of Milk Composition. Academic Press, San Diego, CA.

Johansson, E. M., S. A. Crusz, E. Kolomiets, L. Buts, R. U. Kadam, M. Cacciarini, K. M. Bartels, S. P. Diggle, M. Camara, P. Williams, R. Loris, C. Nativi, F. Rosenau, K. E. Jaeger, T. Darbre, and J. L. Reymond. 2008. Inhibition and dispersion of Pseudomonas aeruginosa biofilms by glycopeptide dendrimers targeting the fucose-specific lectin LecB. Chem. Biol. 15:1249-1257.

Kunz, C., and B. Lonnerdal. 1993. Protein composition of Rhesus monkey milk: Comparison to human milk. Comp. Biochem. Physiol. Comp. Physiol. 104:793-797. 
Lesman-Movshovich, E., and N. Gilboa-Garber. 2003. Pseudomonas aeruginosa lectin PA-IIL as a powerful probe for human and bovine milk analysis. J. Dairy Sci. 86:2276-2282.

Lim, I. W., P. J. Stride, R. L. Horvath, C. R. Hamilton-Craig, and P. P. Chau. 2009. Chromobacterium violaceum endocarditis and hepatic abscesses treated successfully with meropenem and ciprofloxacin. Med. J. Aust. 190:386-387.

Long, J., O. Zaborina, C. Holbrook, A. Zaborin, and J. Alverdy. 2008. Depletion of intestinal phosphate after operative injury activates the virulence of $P$. aeruginosa causing lethal gut-derived sepsis. Surgery 144:189-197.

Mitchell, E., C. Houles, D. Sudakevitz, M. Wimmerova, C. Gautier, S. Perez, A. M. Wu, N. Gilboa-Garber, and A. Imberty. 2002 Structural basis for oligosaccharide-mediated adhesion of Pseudomonas aeruginosa in the lungs of cystic fibrosis patients. Nat. Struct. Biol. 9:918-921.

Morin, D. E., L. L. Rowan, W. L. Hurley, and W. E. Braselton. 1995 Composition of milk from llamas in the United States. J. Dairy Sci. 78:1713-1720.

Naarding, M. A., I. S. Ludwig, F. Groot, B. Berkhout, T. B. Geijtenbeek, G. Pollakis, and W. A. Paxton. 2005. Lewis X component in human milk binds DC-SIGN and inhibits HIV-1 transfer to CD4 T lymphocytes. J. Clin. Invest. 115:3256-3264.

Newburg, D. S. 2009. Neonatal protection by an innate immune system of human milk consisting of oligosaccharides and glycans. J. Anim. Sci. 87:26-34.

Newburg, D. S., S. Ashkenazi, and T. G. Cleary. 1992. Human milk contains the Shiga and Shiga-like toxin receptor glycolipid Gb3. J. Infect. Dis. 166:832-836.

Perret, S., C. Sabin, C. Dumon, M. Pokorna, C. Gautier, O. Galanina, S. Ilia, N. Bovin, M. Nicaise, M. Desmadril, N. Gilboa-Garber, M. Wimmerova, E. P. Mitchell, and A. Imberty. 2005. Structural basis for the interaction between human milk oligosaccharides and the bacterial lectin PA-IIL of Pseudomonas aeruginosa. Biochem. J. 389:325-332.

Ruiz-Palacios, G. M., L. E. Cervantes, P. Ramos, B. ChavezMunguia, and D. S. Newburg. 2003. Campylobacter jejuni binds intestinal $\mathrm{H}(\mathrm{O})$ antigen (Fuc alpha 1, 2Gal beta 1, 4GlcNAc), and fucosyloligosaccharides of human milk inhibit its binding and infection. J. Biol. Chem. 278:14112-14120.
Schleiss, M. R. 2006. Role of breast milk in acquisition of cytomegalovirus infection: Recent advances. Curr. Opin. Pediatr. 18:48-52.

Sirinavin, S., C. Techasaensiri, S. Benjaponpitak, R. Pornkul, and M. Vorachit. 2005. Invasive Chromobacterium violaceum infection in children: Case report and review. Pediatr. Infect. Dis. J. 24:559 561.

Strateva, T., and D. Yordanov. 2009. Pseudomonas aeruginosa-A phenomenon of bacterial resistance. J. Med. Microbiol. 58:11331148.

Strömqvist, M., P. Falk, S. Bergström, L. Hansson, B. Lonnerdal, S. Normark, and O. Hernell. 1995. Human milk kappa-casein and inhibition of Helicobacter pylori adhesion to human gastric mucosa. J. Pediatr. Gastroenterol. Nutr. 21:288-296.

van Hoffen, E., B. Ruiter, J. Faber, L. M'Rabet, E. F. Knol, B. Stahl, S. Arslanoglu, G. Moru, A. Boehm, and J. Barssen. 2008. A specific mixture of short-chain galacto-oligosaccharides and longchain fructo-oligosaccharides induces a beneficial immunoglobulin profile in infants at high risk for allergy. Allergy 64:484-487.

Vos, A. P., L. M'Rabet, B. Stahl, G. Boehm, and J. Garssen. 2007. Immune-modulatory effects and potential working mechanisms of orally applied nondigestible carbohydrates. Crit. Rev. Immunol. $27: 97-140$

Wilson, N. L., L. J. Robinson, A. Donnet, L. Bovetto, N. H. Packer, and N. G. Karlsson. 2008. Glycoproteomics of milk: Differences in sugar epitopes on human and bovine milk fat globule membranes. J. Proteome Res. 7:3687-3696.

Wu, A. M., J. H. Wu, T. Singh, J. H. Liu, M. S. Tsai, and N. GilboaGarber. 2006. Interactions of the fucose-specific Pseudomonas aeruginosa lectin, PA-IIL, with mammalian glycoconjugates bearing polyvalent Lewis(a) and $\mathrm{ABH}$ blood group glycotopes. Biochimie 88:1479-1492.

Zinger-Yosovich, K., D. Sudakevitz, A. Imberty, N. C. Garber, and N. Gilboa-Garber. 2006. Production and properties of the native Chromobacterium violaceum fucose-binding lectin (CVIIL) compared to homologous lectins of Pseudomonas aeruginosa (PA-IIL) and Ralstonia solanacearum (RS-IIL). Microbiology 152:457-463. 